\title{
Vamos falar sobre ciência: aproximação da ciência na prática do profissional de educação física na residência multiprofissional
} \author{
in the multidisciplinary residence

\section{Danilo Fernandes da Silva ${ }^{1}$, Marcos Roberto Queiroga ${ }^{2}$} \\ ${ }^{1}$ University of Ottawa (uOttawa), Ottawa/ON, Canadá \\ 2 Universidade Estadual do Centro-Oeste (Unicentro), Guarapuava/PR, Brasil
}

Let's talk about science: approximating science and practice of exercise professionals

\section{HISTÓRICO DO ARTIGO}

Recebido: 16 dezembro 2019 Revisado: 07 julho 2020

Aprovado: 22 julho 2020

\section{PALAVRAS-CHAVE:}

Atenção primária à saúde; Exercício; Saúde Pública; Internato não Médico; Centros de Saúde.

\section{KEYWORDS:}

Primary Health Care; Exercise; Public Health; Non-medical Internship; Health Centers.

\section{RESUMO}

OBJETIVO: Contextualizar o uso das ferramentas metodológicas e relatar as experiências com a disciplina de "Avaliação e Prescrição de Atividade Física para a Saúde e Doença" ministrada para profissionais/residentes de Educação Física (EF).

MÉTODOS: O artigo apresenta as descrições de atividades desenvolvidas para dois profissionais da primeira turma de residência multiprofissional da Universidade Estadual do Centro-Oeste (Unicentro-PR). Buscouse inovar em aspectos do ensino, utilizando como referência metodológica a atividade física baseada em evidências e aprendizagem baseada em problemas.

RESULTADOS: Entre as experiências destacadas estão a importância da busca por referencial teórico e os momentos de aplicação dos conteúdos na prática, e a contextualização dos ensaios pragmáticos fomentando reflexões sobre a prática no âmbito da atenção primária à saúde (APS).

CONCLUSÃO: Espera-se que esse relato propicie a discussão continuada nos cursos de residência multiprofissional sobre a otimização da aplicação da ciência na prática dos profissionais de EF no contexto da APS.

\section{ABSTRACT}

OBJECTIVE: To contextualize the use of methodological tools and report the experiences with the course "Assessment and Prescription of Physical Activity for Health and Disease" ministered for professionals/residents in Physical Education (PE).

METHODS: The article presents the descriptions of activities developed for two professionals of the first class from the multidisciplinary residence of the Midwestern Parana State University (Unicentro-PR). We aimed to innovate in teaching aspects, using methodological references such as evidence-based physical activity and program-based learning.

RESULTS: There are some highlighted experiences such as the importance of searching for the theoretical reference and the timing to apply the topics, optimizing the application of science in the residences' practice and the pragmatic trials stimulating evidences that reflects practices.

CONCLUSION: We hope this report provides the continuous discussion on multidisciplinary residence courses about the optimization of science applied into practice by PE professionals in the primary health care context. 


\section{INTRODUÇÃO}

A relação entre Educação Física (EF) e saúde pública tem se tornado cada vez mais forte, especialmente a partir da criação do Núcleo de Apoio à Saúde da Família (NASF), pela Portaria no 154 , de 24 de janeiro de 2008. Esta portaria inseriu formalmente o profissional de EF na Atenção Primária à Saúde (APS) (BRASIL, 2008). Esta interação entre as áreas vem sendo consolidada com a oferta de disciplinas específicas em nível de graduação (LOCH et al., 2016; DA SILVA et al., 2019) e da criação de residências multiprofissionais, que contribuem com o aprimoramento teórico-prático intensivo no contexto do Sistema Único de Saúde (SUS) (LOCH et al., 2012).

Em 2015, a residência multiprofissional em atenção primária com ênfase em saúde da família foi aprovada para implantação no Setor de Ciências da Saúde da Universidade Estadual do Centro-Oeste (UNICENTRO-Guarapuava-PR). O primeiro edital do programa foi publicado para seleção em 2016. Anualmente, são selecionados oito profissionais, dois de cada área, correspondentes à EF, Nutrição, Fisioterapia e Enfermagem. Destaca-se que o objetivo geral do programa de residência multiprofissional é formar profissionais para atuar na Saúde da Família, na concepção multiprofissional, por meio da assistência humanizada e integral, com foco na promoção de saúde e nos modelos de atenção, baseados nos princípios e diretrizes do SUS. Possui duração de quatro semestres, com carga horária de $5.760 \mathrm{~h}$, das quais $1.152 \mathrm{~h}$ de teoria (disciplinas gerais e específicas) e $4.608 \mathrm{~h}$ de prática em serviço.

Entre as atividades teóricas estão quatro disciplinas específicas de cada área (uma em cada semestre). Uma das disciplinas específicas para a EF é "Avaliação e Prescrição de Atividade Física para a Saúde e Doença", que possui carga horária de 40h. É ofertada para os residentes do segundo ano (R2), no quarto semestre do programa, com dois encontros mensais de $4 \mathrm{~h} /$ aula cada, durante cinco meses. Este formato possibilita interação dos aspectos teóricos discutidos na disciplina (imersão) com a prática profissional (dispersão).

Embora a maioria dos egressos em EF tenham realizado disciplinas de avaliação e prescrição de exercícios, poucos tiveram a oportunidade de aplicar esses conhecimentos com populações especiais, que representam uma grande parcela dos pacientes na APS. Ainda, a implementação de recomendações "clássicas" apenas norteiam a prescrição de exercícios físicos para populações especiais (e.g., doenças e condições crônicas) (ACSM, 2018; PEDERSEN; SALTIN, 2015; LUAN et al., 2019). Para os cenários que os Residentes em EF irão se deparar na APS, estas recomendações (i.e., prescrição pelos princípios Frequência, Intensidade, Tipo, Tempo focando melhoria geral da saúde) são parâmetros base para o planejamento de um programa, mas não são o suficiente para pacientes com multimorbidades ou condições não exploradas neste guideline (ACSM, 2018) e artigos diretamente ligados à população de interesse (e.g., ataxia, fibromialgia, sarcopenia, cirrose hepática, diabetes gestacional, declínio cognitivo) se tornam essenciais (PEDERSEN; SALTIN, 2015; LUAN et al., 2019).

Além disso, o atendimento individualizado em domicílio ou na unidade (com ou sem a equipe multiprofissional) é mais um fator que requer a aproximação entre ciência e prática para nor- tear a atuação dos profissionais de EF. Portanto, subsidiar o uso de ferramentas que contribuam para uma prescrição de exercícios na "vida real" (prescrição individualizada de acordo com cada caso clínico) deve ser destacado no programa das disciplinas de residência em EF. Neste sentido, o fomento do conceito de prática baseada em evidências e o método de ensino aprendizagem baseada em problemas podem ser estratégias para melhorar a atuação do profissional de EF na APS. Tentativas de discutir esta abordagem tem sido enfatizada pelo nosso grupo em nível de graduação, tanto no ensino (Da Silva et al., 2019) quanto na extensão (QUEIROGA et al., 2019).

Pensando na possibilidade de interação e inovação, os docentes responsáveis pela disciplina "Avaliação e Prescrição de Atividade Física para a Saúde e Doença" recorreram ao método de ensino associada aos princípios da "aprendizagem baseada em problemas (Problem-based learning)" (MAMEDE, 2001), sendo esta uma sugestão do Ministério da Saúde para os programas de residência (BRASIL, 2006). A aprendizagem baseada em problemas auxilia na construção da ponte entre conhecimento científico e prática profissional e, em suma, considera o que os estudantes já sabem e o que precisam saber para melhorar a qualidade de seu serviço (no caso, a prescrição de exercícios). Alguns princípios desse método de ensino são: i) enfatizar a aprendizagem ativa e centrada no aluno (aprendizagem é centrada nos problemas levantados pelos alunos); ii) capacitar alunos para aprenderem no contexto em que o conhecimento pode ser usado, aumentando a capacidade de aplicar ciência na prática; iii) aprender acrônimos e estratégias para facilitação da aprendizagem (ver PICO abaixo); iv) ensinar ao aluno como monitorar seu próprio progresso e avaliar sua atuação.

Além disso, utilizaram de ferramentas adicionais como: 1) uma estratégia de busca de informações científicas denominada "PICO" (i.e., participantes, intervenção, comparador, outcome); 2) uma alternativa metodológica para o desenho de pesquisas que oferece respostas práticas a questões do "mundo real" no contexto da saúde denominada ensaio pragmático (COX; LEWIS, 2016).

Desse modo, nosso objetivo foi contextualizar o uso das ferramentas metodológicas e relatar as experiências com a disciplina de "Avaliação e Prescrição de Atividade Física para a Saúde e Doença" ministrada para profissionais/residentes de EF

\section{MÉTODOS}

Este trabalho se configura como um relato de experiência referente a ferramentas metodológicas empregadas na disciplina de "Avaliação e Prescrição de Atividade Física para a Saúde e Doença", ministrada para dois profissionais/residentes de Educação Física, pertencentes a primeira turma de residência multiprofissional (2016-2017) da UNICENTRO.

Os resultados são apresentados em quatro experiências de ensino:
1) Apresentação do conceito de ensaio pragmático;
2) Documentário "O renascimento do parto (2013) - Erica de Paula e Eduardo Chauvet, Brasil";
3) A busca do referencial teórico;
4) O contraste da melhor evidência com a evidência disponível 


\section{RESULTADOS}

O modelo lógico da disciplina é exibido na Figura 1. Contextualização e suporte para elaboração das experiências no contexto da "Avaliação e prescrição de atividade física para a saúde e doença".

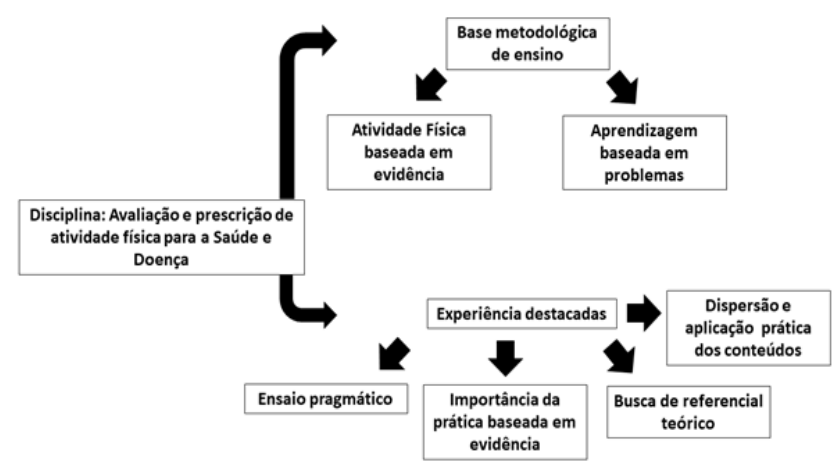

Figura 1. Modelo organizacional da disciplina "Avaliação e prescrição de atividade física para a Saúde e Doença".

Em 2010, um dos professores da disciplina teve contato, através do Programa de Educação Tutorial (PET), com estudantes de doutorado canadenses que eram parte de um programa apoiado pelo governo de seu país denominado "Let's talk science" - Vamos falar sobre ciência. Brevemente, o Let's talk science e seus apoiadores reconhecem que o conhecimento científico é importante para que se possa compreender e participar de mudanças no mundo e é fundamental para construção de uma sociedade inovadora. Segundo eles, "a ciência nos permite questionar o mundo ao nosso redor" (LTS, 2019). A missão do Let's talk science serviu de inspiração para a Experiência 1 - Documentário "O renascimento do parto (2013) - Erica de Paula e Eduardo Chauvet, Brasil"). A ideia era contrapor práticas em saúde muitas vezes ritualísticas e pouco baseadas em evidências científicas com a ideia de uma prática baseada em ciência.

Partindo desse estímulo, os docentes buscaram lançar mão de ferramentas de ensino que propiciassem a aproximação entre conhecimentos científicos e prática de profissionais de EF voltados à saúde, sobretudo para prescrição de atividade física em diferentes condições ou populações. As Experiência 2 - A busca de referencial teórico e Experiência 3 - O contraste da melhor evidência com a evidência disponível buscaram nortear os alunos na aprendizagem de métodos e ferramentas práticas e acessíveis de busca de evidências científicas de qualidade (Experiência 2), bem como treiná-los no estabelecimento de um paralelo entre o que a ciência sugere e o nível de evidência dessa sugestão para que pudessem otimizar suas decisões práticas na seleção, por exemplo, da intensidade de esforço, duração da sessão e tipo de exercício (Experiência 3). Esse exercício vem sendo construído por nosso grupo de estudos e pesquisa no nível de graduação e a experiência tem ajudado os alunos a usarem melhor recomendações científicas em seus estágios (QUEIROGA et al., 2019).

Por fim, utilizou-se como ferramenta de ensino o conceito de ensaios pragmáticos (experiência 4). A ideia de trazer os princípios dos ensaios pragmáticos surgiu de discussões prévias com os residentes em momentos de tutoria (Docentes, Tutores, Preceptores), em que frequentes dúvidas estavam associadas a aspectos da avaliação e prescrição de exercícios para populações especiais. Em geral, dificuldades de prescrever e orientar exercícios físicos para pacientes com doenças crônicas mais presentes na prática (e.g., obesidade, hipertensão, dislipidemia, diabetes tipo 2, lombalgia). Adicionado a isso, o agravante de pacientes que apresentam uma combinação de doenças (multimorbidades) ou outras doenças e alterações menos comuns (e.g., hanseníase, colostomia, urostomia, HIV).

Além disso, as condições de trabalho, infraestrutura, contato com a equipe multiprofissional, disponibilidade de materiais, o grau de incapacidade (pacientes que recebiam atendimento em casa) e adesão dos pacientes dificultavam a implementação de recomendações "clássicas" de avaliação e prescrição de exercícios (e.g., grupos de atividade física nas Unidades de Saúde). Para destacar a importância de uma prescrição de exercícios que respeite as características e potenciais contra-indicações que algumas populações especiais tem, considera-se importante as revisões de Pedersen e Saltin (2015) e Luan et al. (2019). Nesses estudos, aproximadamente 30 doenças ou condições crônicas são revisadas e recomendações gerais de prescrição de exercícios são feitas reforçando a complexidade de atuar com esses grupos populacionais.

Todas as quatro experiências relatadas aqui foram aplicadas sob a perspectiva do método de ensino da aprendizagem baseada em problemas. Este método tornou-se uma valiosa opção, sobretudo porque as discussões continuadas que os profissionais de EF tem dentro da equipe multiprofissional e de problemas oriundos da prática em serviço se assemelham com a proposta dessa metodologia de ensino (e.g., aprendizagem ativa, centrada no aluno, visando capacitá-lo no entendimento do contexto em que o conhecimento pode ser usado, aumentando a capacidade de aplicar ciência na prática) (MAMEDE, 2001).

Experiência 1 - Documentário "0 renascimento do parto (2013) Erica de Paula e Eduardo Chauvet, Brasil"

Antes de apresentar ferramentas e estratégias para busca de referencial teórico, foi utilizado este documentário para reforçar a importância da prática de saúde baseada em evidências. Trechos desse material que reforçam a prática "ritualística" de obstetras e ginecologistas que pouco usam conhecimentos técnico-científicos em sua prática e ficam presos a "hospitalização" do parto foram utilizados.

Ao buscar um contraste com a EF voltada à saúde, foi possível notar como em muitos casos profissionais de EF também acabam se tornando reféns desses "rituais" (e.g., formas padronizadas de se realizar o aquecimento, parte principal e volta à calma, "receitas" de prescrição de exercícios, muitas vezes sem considerar as necessidades individuais do paciente) que engessam a prática e criam dificuldades em fazer uso de conhecimentos científicos (atividade física baseada em evidências). Isso se dá, em alguns casos, por não saberem identifica-los, interpreta-los e aplica-los na prática. No entanto, verificou-se que, ao lidarem com a saúde e, sobretudo com a doença e a incapacidade, os residentes expressaram maior receio de cometerem equívocos nas suas decisões (i.e., não melhorar ou piorar o quadro clínico do paciente) o que aparentemente conferiu abertura maior às informações de rigor científico. 


\section{Experiência 2 - A busca de referencial teórico}

Foi usada a vídeo-aula do Prof. Leonardo Paixão do Centro de Telessaúde do Hospital das Clínicas da Universidade Federal de Minas Gerais sobre prática de saúde baseada em evidências - estratégia de busca. Os residentes foram orientados a inicialmente estabelecer uma pergunta antes da busca, fazendo uso da estratégia PICO (paciente, intervenção, comparação, outcome [desfecho]). Em seguida, apresentaram casos de pacientes seguindo o Problem-based learning e usaram a estratégia PICO nos exemplos de uma paciente com hipertensão arterial e uma com colostomia.

Foi discutido e praticado o trecho da vídeo-aula que sugere que o uso de menos letras (que podemos chamar de conceitos) da estratégia PICO poderia resultar em uma busca mais sensível, enquanto que o uso dos quatro conceitos levaria a uma busca mais específica e restrita, o que muitas vezes faria com que referências úteis não fossem captadas.

A seguir, a vídeo-aula enfocou em tipos de estudos que podemos encontrar e a pirâmide do nível de evidência (AGORITSAS et al., 2014) (Figura 2), indo das evidências com maior risco de viés (pesquisas "In Vitro", estudos com animais) até as de menor risco (ensaios clínicos randomizados, meta-análises). Também foi apresentada a pirâmide de fontes de evidência que parte do menor nível que combina uma única pergunta (i.e., um estudo), passando pelas sinopses de estudos, sínteses, sinopses de sínteses e a melhor fonte de evidência que são os sumários (e.g., guidelines) (DICENSO; BAYLEY; HAYNES, 2009) (Figura 2).

Foram demonstradas as possibilidades de busca de sumários a partir do "National Guideline Clearinghouse", os sumários "Point of care" (e.g., UpToDate, DynaMed, BestPractice) e o tradicional pubmed/MEDLINE. Explorou-se com os residentes em um primeiro momento o pubmed/MEDLINE, considerando que elas já tinham conhecimentos prévios sobre ele.

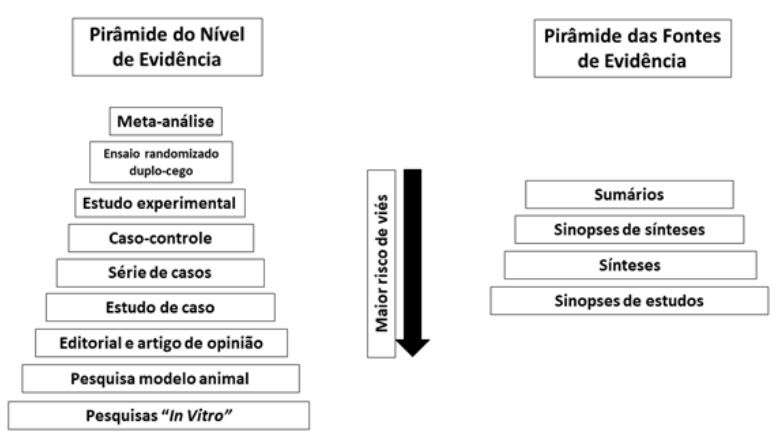

Figura 2. Pirâmides do nível de evidência e das fontes de evidência. Adaptada de: Agoritsas et al. (2014), DiCenso et al. (2009) e da vídeo-aula do Prof. Leonardo Paixão do Centro de Telessaúde do Hospital das Clínicas da Universidade Federal de Minas Gerais.

(link: https://www.youtube.com/watch?v=OczVsxTVEsU\&t=241s).

Experiência 3 - 0 contraste da melhor evidência com a evidência disponível

A busca mais sensível combinando "Paciente" e "Intervenção" na base de dados pubmed/MEDLINE levou a um nível de evidência muito alto para o caso da hipertensão $(P=$ hypertension; I = exercise) (MALACHIAS et al., 2016) e um muito baixo para o caso da colostomia ( $\mathrm{P}=$ colostomy; $\mathrm{I}$ = exercise) (WISKEMANN et al., 2016), o que era esperado. O estudo relacionado à hipertensão que os residentes selecionaram após a busca foi o capítulo 6 da 7a Diretrizes Brasileiras de Hipertensão Arterial (exemplo de sumário), enquanto que o estudo relacionado à colostomia foi um estudo de caso com um paciente em situação similar, que foi avaliado após 2, 4, 6 e 9 meses de intervenção com exercícios combinados (WISKEMANN et al., 2016). Além desses estudos, outros também foram selecionados para leitura e extração de informações que pudessem auxiliá-los na organização da avaliação e prescrição dos exercícios físicos.

Os residentes puderam observar na prática como algumas condições de saúde ainda não apresentam guidelines específicos já que a quantidade/qualidade de estudos ainda é baixa, dificultando a elaboração de recomendações com alto grau de evidência. Ainda assim, notaram ser possível identificar exemplos que podem ser adaptados a sua realidade de trabalho a partir de resultados obtidos em experimentos prévios.

Após realizarmos esse exercício com os residentes, obteve-se seu feedback, o qual foi ressaltado que, de fato, a prática poderia ser mais baseada em evidências. Adicionalmente, muitas de suas dúvidas e receios de causar algum efeito adverso em um determinado paciente poderiam ser minimizados se buscassem aplicar uma intervenção de exercícios físicos baseada em evidências considerando o "pragmatismo" de suas rotinas de trabalho.

\section{Experiência 4 - Apresentação do conceito de ensaio pragmático}

Ao longo do contato com os residentes, notou-se a necessidade de trazer elementos que os auxiliassem nas difíceis decisões que tomam em relação à avaliação e prescrição de exercícios físicos de modo a torna-las mais baseadas em evidências, o que maximizaria as chances de sucesso da intervenção. Assim, foi apresentado o artigo de Cox e Lewis (2016), publicado no Journal of American Medical Association (JAMA) na sessão de "Guia estatístico e metodológico - revisão clínica e educação". Inicialmente, foram verificados os conhecimentos prévios dos residentes em relação ao conceito de ensaio pragmático e notou-se que os profissionais não possuíam definição clara sobre o assunto. Na apresentação, foi lido e debatido este artigo visando demonstrar que há autores que discutem a relevância dos ensaios pragmáticos em relação aos ensaios explanatórios (i.e., ensaios clínicos randomizados), haja vista que a realidade de muitos contextos não permite a aplicação de procedimentos considerados metodologicamente "ideais" e reforçam a necessidade de pesquisadores pensarem alternativas para o "mundo real". Os residentes compreenderam que esses autores estão pensando nas dificuldades de pessoas como eles.

Foi apresentado como as estratégias de ensaios pragmáticos contrastam com os ensaios explanatórios de modo a apresentar resultados com maior aplicação prática (validade externa). Por exemplo, os ensaios pragmáticos costumam dar liberdade a intervenções secundárias à que é foco do estudo e não buscam estratégias para maximizar a adesão dos participantes. Os ensaios explanatórios trabalham com a probabilidade de que a intervenção causa os resultados do estudo, e não outros fatores (validade interna). 
Tunis et al. (2003) apresentaram quatro características dos ensaios pragmáticos que positivamente chamaram a atenção dos residentes: 1) selecionam alternativas clinicamente relevantes para solucionar o problema; 2 ) incluem uma diversidade populacional entre os participantes do estudo; 3) recrutam participantes de cenários práticos heterogêneos e; 4) coletam dados de uma variedade de parâmetros de saúde. Apesar dos esclarecimentos dessa experiência em aula, um residente questionou onde poderiam encontrar informações de ensaios pragmáticos para ajuda-los nas decisões práticas.

Neste momento, foi esclarecido aos residentes que os ensaios pragmáticos têm potencial para ajudá-los nas decisões práticas e foram propostos a mais de 50 anos (SCHWARTZ; LELLOUCH, 1967), mas provavelmente não iriam encontrar soluções para suas dúvidas focando em buscas que enfatizassem o termo "pragmático". Segundo as conclusões de Cox e Lewis (2016), "explanatório" e "pragmático" marcam os extremos de um espectro de desenhos de pesquisa, e, na prática, um estudo apresenta características que o definem como exploratório e outras que denotam pragmatismo. A "Take home message" era de que informações científicas são produzidas frequentemente para ajudá-los na rotina de trabalho. Faltaria naquele momento um aprofundamento de como buscar essas informações de forma efetiva.

\section{CONCLUSÃO}

Apesar da possibilidade de realizar uma residência em atenção primária ser algo relativamente recente ao profissional de $E F$, seu potencial de fortalecimento da área e contribuição com sua inserção e valorização profissional na saúde pública são notáveis. Sendo essa a primeira turma, acredita-se que ainda haverá uma grande evolução do processo de ensino nas residências multiprofissionais, entretanto, é de se ressaltar a relevância das metodologias ativas, buscando aproximar cada vez mais os momentos em sala de aula com a prática profissional. A partir do relato de experiências com os residentes, é possível notar grande potencial da metodologia da aprendizagem baseada em problemas com a adaptação de ferramentas e estratégias que complementam a compreensão e aplicação da especificidade da atividade física baseada em evidência para o profissional de EF no contexto da APS. A ideia de aproximar pesquisa e prática foi relatada com sucesso em outras áreas dentro da EF, como na atuação de professores com características de "pesquisadores" na educação básica, de modo que esses professores trabalham de forma diferenciada e tem grande capacidade auto-formadora, ou seja, é um agente ativo dentro de sua formação continuada (OLIVEIRA et al., 2019).

Ressalta-se também que essa disciplina foi inicialmente programada pela coordenação da residência para ser ministrada no último semestre da especialização. Porém, ao passarem por essa experiência ligada à avaliação e prescrição de exercícios físicos baseados em evidência com o respaldo dos docentes da disciplina, as profissionais repararam o potencial de uso dessas ferramentas para contribuir com sua prática e sugeriram que a disciplina passasse para o primeiro semestre (R1), buscando ter maior tempo para o uso dessas estratégias.

Por fim, o presente relato não "cria" uma nova forma ou modelo mais adequado de se ensinar, apenas sugere alternativas para estimular a aproximação da ciência na prática dos profissionais de EF, nesse caso, daqueles que atuam na APS.

\section{REFERÊNCIAS}

AMERICAN COLLEGE OF SPORTS MEDICINE. Diretrizes do ACSM para os testes de esforço e sua prescrição. 10. ed. Rio de Janeiro: Guanabara Koogan, 2018

AGORITSAS, T.; ISERMAN, E.; HOBSON, N.; COHEN, N.; COHEN, A.; ROSHANOV, P. S.; ... ; HAYNES, R.B. Increasing the quantity and quality of searching for current best evidence to answer clinical questions: protocol and intervention design of the MacPLUS FS Factorial Randomized Controlled Trials. Implementation Science, Manchester, v. 9, p. 125-40, 2014.

BRASIL. Ministério da Saúde. Portaria no. 154 de 24 de janeiro de 2008 Cria os Núcleos de Apoio a Saúde da Família - NASF. Brasília, Governo Federal: Ministério da Saúde, 2008. Disponível em: <http://189.28.128.100/ dab/docs/legislacao/portaria154 2401 08.pdf>. Acessado em: 14 de dezembro de 2019.

BRASIL. Ministério da Saúde. Secretaria de Gestão do Trabalho e da Educação na Saúde. Departamento de Gestão da Educação na Saúde. Residência multiprofissional em saúde: experiências, avanços e desafios. Ministério da Saúde, Secretaria de Gestão do Trabalho e da Educação na Saúde, Departamento de Gestão da Educação em Saúde. Brasília: Ministério da Saúde, 2006. Disponível em: <http://bvsms.saude.gov.br/bvs/publicacoes/ residencia multiprofissional.pdf>. Acessado em: 14 de dezembro de 2019.

COX, H. C.; LEWIS, R. J. Pragmatic trials: practical answers to "real world" questions. Journal of American Medical Association, Chicago, v. 316, n. 11, p. $1205-6,2016$

DA SILVA, D. F.; COUTINHO, S. S.; PICCININI-VALLIS, H.; QUEIROGA, M. R. Physical education in Primary Health Care: reports on interactive actions in an undergraduate course. Revista Brasileira de Atividade Física e Saúde, Florianópolis, v. 23:e0048, 2018

DICENSO, A.; BAYLEY, L.; HAYNES, R. B. Assessing pre-appraised evidence: fine-tuning the $5 \mathrm{~S}$ model into a $6 \mathrm{~S}$ model. Evidence-based Nursing, London, v. 12, n. 4, p. 99-101, 2009.

LET'S TALK SCIENCE. Why STEM? In: Let's Talk Science website. Canada, 2019. Disponível em: <http://letstalkscience.ca/>. Acessado em: 14 de dezembro de 2019.

LOCH, M. Abordando Saúde Coletiva no curso de bacharelado em educação física: relato de experiência. Revista Brasileira de Atividade Física e Saúde Florianópolis, v. 21, n. 3, p. 285-90, 2016.

LOCH, M.; FLORINDO, A. A educação física e as residências multiprofissionais em saúde. Revista Brasileira de Atividade Física e Saúde, Florianópolis, v. 17, n. 2, p. 81-2, 2012

LUAN, X.; TIAN, X.; ZHANG, H.; HUANG, R.; LI, N.; CHEN, P.; WANG, R. Exercise as a prescription for patients with various diseases. Journal of Sport and Health Science, Shanghai, v. 8, n. 5, p. 422-441, 2019.

MALACHIAS, M. V. B.; FRANCO, R. J.; FORJAZ, C. L. M.; PIERIN, A. M. G.; GOWDAK, M. M.; KLEIN, M. R. S. T.; MATSUDO, V. 7th Brazilian Guideline of Arterial Hypertension: Chapter 6 - Non-pharmacological treatment. Arquivos Brasileiros de Cardiologia, Rio de Janeiro, v. 107, Suppl. 3, p. 30 4, 2016.

MAMEDE, S. Aprendizagem baseada em problemas: características, processos e racionalidade. In: MAMEDE, S.; PENAFORTE, J. (Org.) Aprendizagem baseada em problemas: anatomia de uma nova abordagem educacional. Fortaleza: Hucitec, 2001. p. 25-48.

OLIVEIRA, A. T. C.; ROCHA, L. L.; VENÂNCIO, L. SANCHES NETO, L. Professores(as)-pesquisadores(as) de educação física na educação básica: idiossincrasias e fomento à formação na região metropolitana de Fortaleza. Caderno de Educação Física e Esporte, Marechal Cândido Rondon, v. 17, n. 2, p. 143-51, 2019.

PEDERSEN, B. K.; SALTIN, B. Exercise as medicine - evidence for prescribing exercise as therapy in 26 different chronic diseases. Scandinavian Journal of Medicine and Science in Sports, Oslo, v. 25, Suppl. 3, p. 1-72, 2015.

QUEIROGA, M. R.; FERREIRA, S.A; VAZ, E. S.; SOUZA, S. C. S.; OLIVEIRA, L. E. C.; STAVINSKI, N. G. L.; FERNANDES, D. Z.; WEBER, V. R. M.; SILVA, D. F. Clínica e academia escola de educação física: prescrição de exercícios físicos baseados em evidências científicas. Revista de Extensão, Florianópolis, v. 16, p. 111-22, 2019. 
SCHWARTZ, D.; LELLOUCH, J. Explanatory and pragmatic attitudes in therapeutical trials. Journal of Chronic Disease, Washington, v. 20, n. 8, p. 637-48, 1967.

TUNIS, S. R.; STRYER, D. B.; CLANCY, C. M. Practical clinical trials: increasing the value of clinical research for decision making in clinical and health policy. Journal of American Medical Association, Chicago, v. 290, n. 12, p. 1624-32, 2003.

WISKEMANN, J.; SCHOMMER, K.; JAEGER, D.; SCHARHAG-ROSENBERGER, F. Exercise and cancer: return to work as a firefighter with ostomy after rectal carcinoma - a case report. Medicine, Baltimore, v. 95, n. 29, p. e4309, 2016.

\section{AGRADECIMENTOS}

Os autores agradecem ao Programa de Residência Multiprofissional em Atenção Primária à Saúde com ênfase em Saúde da Família da Universidade do Centro-Oeste pelo suporte.

\section{CONFLITO DE INTERESSE}

Os autores do estudo declaram não haver conflito de interesses.

\section{FINANCIAMENTO}

Este estudo não teve apoio financeiro.

\section{ORCID E E-MAIL DOS AUTORES}

Danilo Fernandes da Silva (Autor Correspondente)

ORCID: 0000-0002-4170-1079.

E-mail: danilofernandesdasilva@hotmail.com

Marcos Roberto Queiroga

ORCID: 0000-0002-9284-976X.

E-mail: queirogamr@hotmail.com 